\title{
Breed Differences in Domestic Dogs' (Canis familiaris) Comprehension of Human Communicative Signals
}

\section{Citation}

Wobber, Victoria, Brian Hare, Janice Koler-Matznick, Richard Wrangham, and Michael Tomasello. 2009. Breed differences in domestic dogs' (Canis familiaris) comprehension of human communicative signals. Interaction Studies 10(2): 206-224.

\section{Published Version}

doi:10.1075/is.10.2.06wob

\section{Permanent link}

http://nrs.harvard.edu/urn-3:HUL.InstRepos:5227784

\section{Terms of Use}

This article was downloaded from Harvard University's DASH repository, and is made available under the terms and conditions applicable to Open Access Policy Articles, as set forth at http:// nrs.harvard.edu/urn-3:HUL.InstRepos:dash.current.terms-of-use\#OAP

\section{Share Your Story}

The Harvard community has made this article openly available.

Please share how this access benefits you. Submit a story.

\section{Accessibility}




\title{
Breed differences in domestic dogs' (Canis familiaris) comprehension of human communicative signals
}

\author{
Victoria Wobber 1,2 \\ Brian Hare 2,3 \\ Janice Koler-Matznick ${ }^{4}$ \\ Richard Wrangham ${ }^{1}$ \\ Michael Tomasello ${ }^{2}$
}

(1) Department of Anthropology, Harvard University, Cambridge, MA 02138, U.S.A.

(2) Max Planck Institute for Evolutionary Anthropology, Leipzig, D-04103, Germany

(3) Department of Biological Anthropology and Anatomy, Duke University, Durham NC 27705 U.S.A.

(4) New Guinea Singing Dog Conservation Society, Central Point, OR 97502, U.S.A.

Corresponding author: Victoria Wobber, Department of Anthropology, 11 Divinity Ave, Cambridge, MA 02138, USA. Tel: +1-617-496-4262. Fax: +1-617-496-8041. wobber@,fas.harvard.edu

Running Head: BREED DIFFERENCES IN CUE-FOLLOWING 


\begin{abstract}
Recent research suggests that some human-like social skills evolved in dogs (Canis familiaris) during domestication as an incidental by-product of selection for "tame" forms of behavior. It is still possible, however, that the social skills of certain dog breeds came under direct selection that led to further increases in social problem solving ability. To test this hypothesis, different breeds of domestic dogs were compared for their ability to use various human communicative behaviors to find hidden food. We found that even primitive breeds with little human contact were able to use communicative cues. Further, "working" dogs (shepherds and huskies: thought to be bred intentionally to respond to human cooperative communicative signals) were more skilled at using gestural cues than were non-working breeds (basenji and toy poodles: not thought to have been bred for their cooperative-communicative ability). This difference in performance existed regardless of whether the working breeds were more or less genetically wolf-like. These results suggest that subsequent to initial domesticating selection giving rise to cuefollowing skills, additional selection on communicative abilities in certain breeds has produced substantive differences in those breeds' abilities to follow cues.
\end{abstract}

Key words: dogs; social cognition; evolution; breed differences; domestication 
Domestic dogs are unusually skilled at reading human social and communicative behaviors when compared to other nonhumans (Cooper et al 2003, Hare and Tomasello 2005, Miklosi et al 2005). Dogs have been shown to spontaneously use a number of communicative cues (e.g. a pointing gesture or gaze), including completely novel behaviors, to find hidden food (Agnetta et al 2000, Hare and Tomasello 1999, Miklosi, 2008, Miklosi et al 1998, Riedel et al 2006, Soproni et al 2001, 2002). When directly compared on the same tasks, dogs are even more skillful at using human social cues than chimpanzees and wolves - though human-reared wolves are able to use such cues (Hare et al 2002, Miklosi et al 2003, Viranyi et al 2008). Although wolves have been shown to be able to be trained to use these cues, this is also the case for many non-human primate species - however, it seems unlikely that either wolves or non-human primates show as extensive flexibility in interpreting these cooperative signals as do domestic dogs (Udell et al, 2008). Studies of dog puppies of various age and rearing history suggest that these abilities do not require extensive exposure to humans in order to develop (Hare et al 2002, Riedel et al 2008). Further, fox kits experimentally selected only to approach a human without fear or aggression were as skillful as age matched dog puppies at using a human pointing gesture. Importantly, the kits from the selected population were more skillful than foxes from a control population (i.e. not selected based on their approach behavior towards humans) (Hare et al 2005). These results suggest that the foxes not only evolved dog-like skills for interpreting human social behaviors as a result of their domestication but also that such behaviors can arise simply as an incidental by-product of selection for reduced reactivity to humans (Hare et al 2002, Hare et al 2005). This implies 
that domestic dogs' abilities to follow human signals may have originated in a similar manner, as a byproduct of selection on tame behavior.

While there is evidence to suggest that cue-following skills arose as a byproduct of selection on emotional reactivity (Hare and Tomasello 2005), it is currently unclear whether additional selection in dogs produced alterations in these abilities according to different breeding criteria. Numerous behavioral differences exist among dog breeds, from distinctions in problem-solving behavior to more general traits such as aggressiveness and reactivity (Christiansen et al 2001, Scott and Fuller 1965, Wilsson and Sundgren 1997). However, a recent paper did not find differences between breeds in a human-focused social behavior, detour-following ability (Pongracz et al 2005). As such, dog breeds may not differ in their abilities to follow human cues to hidden food. It is possible that regardless of breeding criteria, close human interaction has produced increased sensitivity to human cues equally in all breeds. Alternatively, one might expect that breeds selected to interact frequently in a communicative context with humans, such as retrievers or shepherds, should use human signals more skillfully than other breeds. No controlled experiment has yet been performed to test this hypothesis. The present experiments were designed to distinguish whether there are in fact differences between breeds in their cue-following abilities.

In our first experiment, we investigated the cue-following abilities of New Guinea Singing Dogs (NGSDs), a group of dogs thought never to have been bred for their ability to use human signals in any context. In fact, it is suspected that NGSDs, a close relative of the Australian dingo, have had little contact with humans for over 5,000 years (Koler- 
Matznick 2002, Koler-Matznick et al 2003, Vila et al 1997). By comparing NGSDs to a cross-section of breeds representing those which have lived in contact with humans in recent history, we were able to discriminate whether general exposure to humans has caused changes in cue-following ability relative to a breed which has had very little interaction with humans. If this general exposure were not important, with dogs' cuefollowing abilities instead being determined by early selection on emotional reactivity and not altered thereafter, then the NGSDs and the other dog breeds should show similar abilities to follow human cues.

In the second experiment, we aimed to determine whether there are distinctions in cue-following among breeds that have had similar degrees of contact with humans. We investigated two potential hypotheses that might explain changes in cue-following performance. First, we asked whether dogs selected to interact in communicative contexts with humans perform better in tests of cue-following than those not subject to such selection. This would be expected if communicative skill is a heritable trait that has been selected in these breeds. Alternatively, since certain breeds of dog have been demonstrated to be more genetically wolf-like than others (Parker et al 2004), a breed's genetic distance from wolves might explain variation in cue use. This would be expected if communicative skills were enhanced by more general selection pressures on tame behavior rather than by selection for skill in interpreting human signals. In this second experiment we used a $2 \times 2$ design to determine which hypothesis better explained variation in cue-following across breeds.

\section{Experiment 1}


In our first experiment we compared the cue-following abilities of a group of dogs belonging to various breeds to that of New Guinea Singing Dogs (NGSDs). Since both groups are domesticated, we predicted that they would both show some skill at using human social cues. But, based on the difference in intensity of human involvement in their different breeding history, we predicted that the NGSDs would use cues less skillfully or in a different way relative to the other dogs.

\section{Method}

\section{Subjects}

Eighteen dogs from nine different breeds (mean age of 4.6 years with 4 females and 14 males) and nine New Guinea Singing Dogs (mean age of 4.7 years with 5 females and 4 males) participated in this experiment. Individuals were from the following breeds: 6 golden retrievers, 3 labrador retrievers, 3 dalmatians, 2 German shepherds, 1 Bernese mountain dog, 1 Irish setter, 1 hovawart, and 1 German pinscher. Though most of these are considered to be working dogs by major kennel clubs, some are also 'companion' breeds. We used a mix of breeds to represent the average performance of working and companion dogs, rather than singling out any one breed to compare to the NGSDs. The pedigree of the subjects in the working/companion dog group was assured by assuring that the subjects' parents were pure-bred (i.e. registered with a kennel club). All dogs were raised as family pets and were from Leipzig, Germany. All but one of the NGSDs were raised by the third author (J.M.-K.) at the New Guinea Singing Dog Conservation Society in Central Point, Oregon where they are housed together. The ninth was tested at its owner's home. 
NGSDs were only recently described. They represent an ancient lineage closely related to Australian dingos and show characteristic signs of domestication (e.g. dog-like forms of tame behavior toward humans as adults, etc). However, few, if any, NGSDs are kept as pets in New Guinea or elsewhere and it is believed that these dogs have lived virtually free of human contact for thousands of years (Koler-Matznick 2002, KolerMatznick et al 2003). All of the NGSDs in the current study were raised largely in the same way one would raise a pet dog. They were frequently held and played with as puppies and have lived in close contact with humans and other dogs during their adult life.

To ensure that subjects maintained motivation throughout testing owners were asked not to feed their dogs on the morning that they were tested. During testing water was always available to all subjects ad libitum.

Set-up

The NGSDs were tested in a familiar enclosure of their outdoor kennel. The working/companion dogs were tested in a novel room at the Max Planck Institute dog testing facility. Two bowls $(16 \mathrm{~cm}$ diameter, $8 \mathrm{~cm}$ high) were placed on the ground 1.2 meters apart. One experimenter (E1) sat behind the bowls and across from the subject who was held on a leash by another experimenter (E2) at the starting point which was 1.5 meters from each of the bowls (Figure 1).

\section{Procedure}

In order to introduce each subject to the test situation E1 showed the subject a piece of food while E2 held the subject at the starting point. Once E1 had the subject's 
attention (sometimes this required calling the subject's name), E1 would then place the food into one of the two bowls as the subject watched. After a bowl was baited the subject was released by E2 and allowed to approach one of the bowls. If they touched the baited bowl first they were allowed to retrieve and eat the food, while if they chose the empty bowl first they were shown where the food was hidden but not allowed to eat the food.

If subjects retrieved food in 13 or more of 18 of these pretest trials, they moved on to the test conditions. The test procedure was identical to that of the introduction with the exception that once E1 captured the attention of the subject E1 sham-baited one of the bowls while hiding the food in the other (or vice versa) - so that subjects knew the food was hidden but did not know where. Bowls were always baited in full view of the subjects, both in test and control conditions, as performed in previous studies of this type (Agnetta et al 2000, Hare et al 1998). Once the bowl was baited and the subject was attentive, E1 gave one of three social cues to communicate the location of the hidden food.

Point and Gaze. E1 extended her cross-lateral arm and index finger (approximately $50 \mathrm{~cm}$ from the bowl) toward the baited bowl while turning her head and eyes to look in the direction of the bowl.

Block. E1 showed the subject a small wooden block $\left(8 \mathrm{~cm}^{3}\right)$ by shaking it rapidly and then placed it in front of the baited bowl as the subject watched. E1 then took her original position and stared straight ahead. 
Static block. E1 showed the subject the same block as used in the previous condition, then the subject's eyes were covered while E1 placed the block silently in front of the baited bowl. E1 then returned to her original position and the subject's eyes were uncovered. Thus the subject could use the block sitting in front of the correct bowl as a cue, but never saw the block moving toward the baited bowl.

All of the visual cues were available until the subject chose one of the bowls by touching it. Again, if the subject chose the correct bowl it was allowed to eat the food reward, and if it chose the incorrect bowl it was shown the location of the food but not given the food. If at any time during a test session a subject failed to touch one of the two bowls in three consecutive trials, they were given a short break and only resumed testing after they had succeeded in four consecutive pretest trials.

A control condition was also run to ensure that subjects were not able to discern the location of the food from any scent or unintended experimenter cues. This control condition was run with all subjects except three of the NGSDs (time and social constraints prevented them from being tested in the control). In this condition, E1 performed the exact baiting procedure as for the test conditions (sham baiting one bowl and actually baiting the other), then returned to her original position and stared straight ahead. The subject was then released as in the other conditions.

Design

Subjects received eighteen trials of each of the three communicative cues (54 test trials total) and eighteen trials of the control condition for a total of 72 trials. All subjects received the social cues in the same order: Point/Gaze, Block, Static Block, and control 
condition. Thus, this meant that subjects received 18 trials of Point/Gaze, then had a small break, then continued with 18 trials of the Block cue, etc. This order was used because it was believed to be given in increasing difficulty (the Block cue being harder than the Point/Gaze, the Static Block harder than the Block, and the Control condition the hardest). The rationale for this was that this provided subjects with the most possible test experience when they received the harder cues, so that in case there was any confusion with the paradigm this would be overcome upon receiving the more difficult cues. While effects of fatigue may have been present across the conditions, as we mentioned our abort criteria ensured that subjects were continuing to participate and we gave subjects breaks between conditions. Further, our next experiment controlled for any potential order effects that may have been exhibited here.

A bowl was never baited with the food for more than two trials in a row and the location of the food reward was counterbalanced within and across subjects. Bowls were sham baited in the same order for every trial for every subject.

Scoring and Analysis

A camera was placed in the corner of the testing room so that both the experimenter and the subject were always recorded. Subjects' choices were coded live with a choice being scored as soon as the subject touched one of the two bowls (the distance between bowls prevented subjects from touching two bowls simultaneously). The data was analyzed in several ways: the performance of both groups with the three communicative cues and the control cue was compared to chance using a one-sample ttest. In addition, the performance of the two different groups of dogs with each of the 
social cues was compared using a two-way repeated measure ANOVA with breed and social cue as factors, with 2-tailed p-values reported below except where stated otherwise. To test for learning or a loss of motivation within each of the testing sessions, the first nine and last nine trials of each test condition from each of the two test groups were compared using a paired t-test (i.e. learning would lead to more correct choices in the second half of the trials for each condition while a loss of motivation would lead to fewer correct choices). Finally, individual performance was examined by assessing whether a subject performed significantly above chance, in this case being attained if they chose the baited bowl in 13 or more of 18 trials ( $<<0.05$, binomial probability, one tailed). The proportions of these successful individuals in the two groups were compared to each other using a $\chi^{2}$ test to determine whether there were significant differences between the groups in the proportion of individuals successful on each cue.

\section{Results}

Both the working/companion dogs and the NGSDs used all of the different communicative cues provided to find the hidden food significantly above chance levels [working/companion dogs: Point/Gaze $\mathrm{t}(17)=10.15, \mathrm{p}<0.001 ;$ Block $\mathrm{t}(17)=3.94$, $\mathrm{p}=0.001 ;$ Static Block $\mathrm{t}(17)=4.40, \mathrm{p}<0.001$; NGSDs: Point $/$ Gaze $\mathrm{t}(8)=4.26, \mathrm{p}=0.003$; Block $\mathrm{t}(8)=6.69, \mathrm{p}<0.001 ;$ Static Block $\mathrm{t}(8)=2.34, \mathrm{p}=0.047$, one sample t-test; Figure 2]. In contrast, when no visible cue was provided in the control subjects did not find the hidden food above chance levels $[\mathrm{t}(24)=0.666, \mathrm{p}=0.51]$

The repeated measure ANOVA (with Cue $\mathrm{x}$ Breed group as factors) revealed a main effect of cue type $[F(2,24)=9.289, p=0.001]$ and a significant interaction between 
success with the different communicative cues and the two different breed groups [F $(2,24)=12.999, \mathrm{p}<0.001]$, but detected no overall effect of breed group (working/ companion dog vs. NGSD). Post-hoc tests revealed that the effect of cue was due to subjects performing significantly better on the Point/Gaze cue than on the Static Block cue $[p<0.001$, Tukey HSD], and significantly better on the Block cue than on the Static Block cue $[\mathrm{p}=0.04$, Tukey HSD]. The interaction between cue and breed group derived from the working/companion dogs performing significantly better than the NGSDs on the Point/Gaze cue $[p=0.006$, Tukey HSD] while in contrast the NGSDs performed significantly better than the working/companion dogs on the Block cue $[\mathrm{p}=0.02$, Tukey HSD]. The two groups did not differ in their use of the Static Block cue $[p=0.70$, Tukey HSD] (see Figure 2). Finally, there was no evidence that the performance of either group with any cue increased with learning, as a paired comparison of subjects' performance on the first and last nine trials of each cue revealed no significant differences [paired sample t-tests, one tailed due to the hypothesis that learning would enhance performance].

Subjects' performance as individuals was consistent with the group analyses. Almost all of the individual dogs used at least one of the cues significantly above chance with fifteen working/companion dogs (83\%) and all of the singing dogs (100\%) using at least one communicative cue successfully [ $p<0.05$, binomial probability]. Meanwhile, eight working/companion dogs (44\%) used multiple cues as individuals but only two singing dogs (22\%) did the same [ $p<0.05$, binomial probability]. Considering the performance of individuals with each of the different cues separately, fourteen working/ companion dogs (78\%) used the Point/Gaze cue while only three singing dogs (33\%) did 
so [p $<0.05$, binomial probability]. Meanwhile, six working/companion dogs $(33 \%)$ and six singing dogs (66\%) used the Block cue as individuals [ $\mathrm{p}<0.05$, binomial probability] while four working/companion dogs (22\%) and three singing dogs (33\%) used the Static Block cue as individuals. These differences in proportion of individuals successful in each group were not significant [ $\chi^{2}$ Fisher's exact test], but still suggest some distinctions between groups in how specific individuals succeed at the task.

\section{Discussion}

Experiment 1 demonstrated that NGSDs were able to follow cues above chance levels. The skill of the NGSDs relative to that of wolves who show little, if any, spontaneous skill at using human cues (Hare et al 2002, Miklosi et al 2003, note the exception for human-reared wolves, Viranyi et al 2008), is particularly noteworthy given the fact that they are thought to be among the domestic dogs who have had the least contact with humans over the course of their evolution. We conclude that cue-following abilities arose early in the course of domestication. In conjunction with the fox results (Hare et al, 2005), this suggests that little selection is required to produce such abilities, given that the foxes acquired cue-following skills after only 40 generations of selection and NGSDs possess these abilities even with a history of only mild domesticating selection. Thus, these results suggest that cue-following abilities were present early on during canid domestication.

NGSDs and the other dogs differed in their performance on the Point/Gaze and the Block cues, in that working/companion dogs performed better than NGSDs on the former while NGSDs outperformed working/companion dogs on the latter. This suggests 
that the two cues reflect different abilities which in turn were affected differentially by recent selection. The enhanced ability of working/companion dogs on the Point/Gaze cue may have been a result of general increased genetic distinction from wolves, potentially resulting from a greater degree of human exposure over the course of their evolutionary history. On the other hand, it is possible that the breeds used in the test were among those directly bred for their ability to read human signals. To discriminate between these possibilities, in Experiment 2 we tested the cue-following skills of breeds varying in their general divergence from wolves and their intensity of selection for cooperativecommunicative abilities.

\section{Experiment 2}

In this experiment we tested four breeds of dogs to discriminate between two potential hypotheses. These two hypotheses were: 1) general exposure to humans and increased distinction from wolves led to augmented cue-following ability; 2) specific selection on communicative ability enabled increased cue-following skills. These two hypotheses are not mutually exclusive. By setting up a 2x2 design we were able to distinguish which of these impacted cue performance to a greater degree.

\section{Method}

Forty-one dogs participated in Experiment 2, separated into four groups based on the factor "working" (whether or not they were from a breed considered to have been selected to cooperate and communicate with humans), and the factor "wolflike" (determined in accordance with the results of Parker et al, 2004). The working breeds selected were those classified as working by the world's four major kennel clubs 
(the Federation Cynologique International, the American Kennel Club, the Kennel Club of the United Kingdom, and the Australia/New Zealand Kennel Club). The genetically wolf-like breeds were chosen from among the nine breeds of dog reported to be significantly more wolf-like than all other breeds of domestic dogs tested (Parker et al 2004). Accordingly, our four different breed groups were as follows:

Less wolf-like working dogs: This group consisted of ten shepherds ( 8 German shepherds and 2 Belgian shepherds). Both of these breeds are less wolf-like genetically (Parker et al 2004) and both are thought to have been selected to use human communicative cues while herding or hunting other animals (Coppinger and Schneider 1995, Scott and Fuller 1965, p. 48). This group was composed of 6 males and 4 females, with a mean age of 3.4 years.

More wolf-like working dogs: This group consisted of twelve Siberian huskies.

Huskies are among the nine breeds that are more wolf-like genetically (Parker et al, 2004) but are also believed to have been selected based on their ability to use communicative cues while transporting humans (Coppinger and Coppinger 2001, p. 49, Coppinger and Schneider 1995). This group was composed of 7 males and 5 females, with a mean age of 5.5 years.

Less wolf-like non-working dogs: This group consisted of ten toy poodles.

Toy poodles are less-wolf like genetically (Parker et al 2004) but are believed to have mainly been bred to be "neat, pretty dogs" (Scott and Fuller 1965, p.46). Given that they are considered difficult to train, we assume that they have been under relatively little selection for their communicative ability (Hart and Hart 
1985). This group was composed of 6 males and 4 females, with a mean age of 5.4 years.

More wolf-like non-working dogs: This group consisted of nine basenji.

The basenji was among the nine breeds found to be more wolf-like genetically (Parker et al 2004). Although they are hunters, they are considered "primitive" sight hounds that chase their quarry without any human intervention (Scott and Fuller 1965, p. 49). There is no evidence suggesting that they have been selected for their ability to communicate with humans. This group was composed of 5 males and 4 females, with a mean age of 5.3 years.

The methods of the current experiment were the same as those used in Experiment 1 with a few exceptions. First, a different object was used in the Block condition (a slightly smaller wooden block painted black and white). Secondly, the Static Block condition was replaced with a Gaze condition in which the experimenter directed her head and eyes toward the correct bowl after it was baited and until the subject chose one of the two bowls. This replacement was made because subjects in both groups succeeded on the Static Block condition in the previous experiment, and we wanted to employ a more difficult condition to better distinguish between groups. Thus we hoped that in the Gaze condition, some groups would succeed and some would not. Finally, the order in which the cues were given was counterbalanced across subjects with the constraint that the control condition was never administered first (to assure motivation in later sessions). Again, 18 trials of each cue were administered.

\section{Results}


Each of the four breeds, as a group, performed significantly above chance on both the Point/Gaze and the Block cues, while only the Shepherds and Basenji performed above chance as a group with the Gaze Cue [one sample t-test, Huskies: Point/Gaze t(11) $=4.87, \mathrm{p}<0.001 ;$ Block $\mathrm{t}(11)=3.807, \mathrm{p}=0.003 ;$ Gaze $\mathrm{t}(11)=1.34, \mathrm{p}=0.21$; Shepherds:

Point/Gaze $\mathrm{t}(9)=5.36, \mathrm{p}<0.001 ;$ Block $\mathrm{t}(9)=4.22, \mathrm{p}=0.002 ;$ Gaze $\mathrm{t}(9)=4.35, \mathrm{p}=0.002$; Basenji: Point/Gaze $\mathrm{t}(8)=3.42, \mathrm{p}=0.009 ;$ Block $\mathrm{t}(8)=3.27, \mathrm{p}=0.01 ;$ Gaze $\mathrm{t}(7)=2.35$, $\mathrm{p}=0.05$; Toys: Point/Gaze $\mathrm{t}(9)=4.21, \mathrm{p}=0.002 ;$ Block $\mathrm{t}(9)=2.86, \mathrm{p}=0.02 ;$ Gaze $\mathrm{t}(8)=$ 0.32, $\mathrm{p}=0.76$; Figure 3]. Despite this mixed success on the Gaze cue, when looking at the cumulative performance on all three cues, each group was also significantly above chance [Huskies: $\mathrm{t}(11)=6.14, \mathrm{p}<0.001$; Shepherds: $\mathrm{t}(9)=5.39, \mathrm{p}<0.001$; Basenji: $\mathrm{t}(7)=$ 4.00, $\mathrm{p}=0.005$; Toys: $\mathrm{t}(8)=7.47, \mathrm{p}<0.001]$. Subjects did not find the hidden food above chance levels in the control condition, where no visual cue was provided $[\mathrm{t}(30)=0.804, \mathrm{p}$ $=0.42]$. The reduced sample size in this instance was because not all subjects completed the control condition - many lost interest and refused to choose between the two options, thus meeting the abort criteria. If anything, this further supports the hypothesis that no unintentional cues were being provided by the experimenter or through olfaction, since such extraneous cues would have led subjects to continue to participate, and perform above chance levels, rather than become frustrated with the test as appeared to be the case.

A repeated measure ANOVA (Cue x Working dog x Wolf-like as factors) detected a main effect of cue type $[\mathrm{F}(2,34)=19.411, \mathrm{p}<0.001]$ and a main effect of the betweensubject factor "working dog" $[\mathrm{F}(1,32)=4.45, \mathrm{p}=0.05]$. Working dogs (shepherds and 
huskies), with a mean of 12.0 correct choices, outperformed non-working dogs (toy poodles and basenji), with a mean of 11.0 correct choices, on their overall use of the three communicative cues, though working dogs did not significantly outperform non-working dogs on any one cue in particular. Post-hoc tests revealed that subjects as a whole performed significantly better on the Point/Gaze cue than on the Gaze cue [p $<0.001$, Tukey HSD], and significantly better on the Block cue than on the Gaze cue $[\mathrm{p}<0.001$, Tukey HSD]. There was no significant difference on performance between the Point/ Gaze and the Block cues $[p=0.87$, Tukey HSD]. Finally, there was no evidence of learning or loss of motivation within test sessions with any of the communicative cues by any of the four different breed groups when the first and last nine trials of each cue were compared [paired sample t-test, one tailed]. A repeated measures ANOVA with Cue and Order as factors showed that there was no effect of the order conditions were presented either on overall performance or on performance on any cue in particular.

Subjects' performance as individuals was consistent with the group analyses. Nineteen of twenty-two (86\%) working dogs and only eleven of nineteen (58\%) nonworking dogs used at least one of the three social cues significantly above chance $[\mathrm{p}<0.05$, binomial probability: Figure 4]. This distinction between the two groups was significantly more than that predicted by chance [ $\chi^{2}$ Fisher's exact test, $\left.\mathrm{p}<0.05\right]$. Moreover, eight working dogs (36\%) used multiple cues as individuals while only two non-working dogs $(11 \%)$ did the same [ $<0.05$, binomial probability: Figure 4]. This result was marginally significant in terms of the working dogs having more individuals succeeding on two cues than the nonworking dogs [ $\chi^{2}$ Fisher's exact test, $\left.\mathrm{p}=0.058\right]$. In 
contrast, there were not significant differences in the percentage of individuals using cues between the wolf-like breeds and non-wolf like breeds [Figure 5]. Comparable numbers of both groups used at least one of the three social cues significantly above chance: fifteen wolf-like dogs (71\%) and fifteen non wolf-like dogs did so (75\%). In turn, six wolf-like dogs (29\%) and four non wolf-like dogs (20\%) used multiple cues above chance levels, indicating that there were no differences between wolf-like and non-wolf like breeds in these measures.

\section{Discussion}

In Experiment 2 all breeds were able to use cues significantly above chance levels, similar to the results of Experiment 1. The results also suggested that selection for communicative ability impacted performance to a greater degree than did genetic distance from wolves, because the working breeds used cues better than the non-working breeds while there were few differences between the wolf-like and non wolf-like groups. At a group level, the huskies and shepherds only averaged one more correct choice overall compared to the basenjis and toys, but in individual performance the differences were more pronounced. Over three times as many working dogs as non-workers used multiple human social cues successfully. While the results of each individual condition were somewhat conflicting, for example with working dogs trending to perform better on the Point/Gaze and Block conditions but not on the Gaze condition, the results of cuefollowing performance overall contradict the hypothesis that the genetic distance from wolves can fully explain the differences in social skill observed across breeds. Instead, the finding that the huskies and shepherds were most skilled at using the social cues 
supports the hypothesis that after domestication, selection acted directly on these breeds' abilities to cooperate and communicate with humans.

\section{General Discussion}

Our findings support the hypothesis that dogs acquired cue-following abilities before the evolution of different breeds. In Experiment 1, both the New Guinea Singing Dogs and the working/companion dogs were skilled at using all three of the social cues, as were the four breed groups tested in Experiment 2. We did not find evidence for any effect of learning in either experiment, as demonstrated by the lack of improvement over the course of multiple trials of each cue. Subjects did not appear to use olfaction or unintentional experimenter cues to find the hidden food as they failed in the control condition when no cue was provided. These results imply that cue-following arose initially as a byproduct of selection on emotional reactivity, as argued for foxes (Hare et al 2005), during the first wave(s) of domesticating selection.

Though all groups tested used the cues above chance levels, there were significant differences in how breeds employed the varying cues presented. Curiously, the NGSDs were the most skilled of any group at using the Block cue. This performance may represent a more primitive form of social cue use. If early dogs were particularly interested in objects manipulated by conspecifics, domesticating selection could have shifted this tendency to an interest in objects manipulated by humans. In turn, early cue use may have been focused on human-manipulated objects, thus accounting for NGSDs' superior performance on the Block cue relative to the Point/Gaze cue. Further selection could then have produced abilities to follow human pointing gestures, leading to the 
superior skills in the Point/Gaze cue demonstrated in the other breeds relative to the NGSDs. Therefore, the current set of comparisons suggests that primitive dogs (similar to the modern NGSD) were skilled at using human social cues as a result of domestication, but over time these skills came under direct selection so that modern dogs are more attuned to human communicative behaviors.

The results of Experiment 2 suggested that this direct selection acted on dogs bred for their abilities to interpret human signals. We found that breeds traditionally bred to fill a communicative role in their interactions with humans, or working breeds, used the social cues differently and in most cases more skillfully relative to non-working breeds. Such distinctions were present regardless of a breed's genetic relation to wolves (Figure 3). Therefore, it is unlikely that the flexibility seen in the working dogs is due to a more general selection pressure, such as for more tame forms of behavior or perhaps even morphological traits. Instead, our results support the hypothesis that communicative skill was selected as an independent trait.

It is possible that the difference in skill between the working and non-working breeds seen here was due not to direct selection on social skill in working breeds, but instead to selection against this skill in non-workers. However, this would imply that working dogs have largely retained the ancestral form of these skills, with non-workers' abilities being selectively reduced. In contrast, the present results suggest that working breeds' cue-following abilities are in fact enhanced relative to those of the ancestral form as represented by the NGSDs. Notably, non-working breeds' performance on the Point/ Gaze cue closely resembles that of the NGSDs, suggesting that without direct selection 
for communicative ability this level of skill has been retained in these breeds. As such, it is likely that positive selection in working breeds led to their improved skills relative to the level seen in NGSDs and non-working breeds.

Since only one breed was used to represent each group in Experiment 2, characteristics specific to that breed rather than typical of that group as a whole could have shaped the present results. For example, perhaps toy poodles cannot be used to represent all genetically non-wolf-like non-working breeds, since this lineage in particular may have been selected differently than other breeds in this category. Yet it seems unlikely that individual quirks of a given breed would have produced the distinctions seen here which followed our hypotheses of selection pressures. In this case it is more parsimonious to conclude that huskies and shepherds showed improved cue performance because of their status as working breeds rather than both breeds being independently selected by chance to demonstrate these skills.

It will be important in future studies to evaluate the effects of rearing history on breed differences in cue-following ability. Dog puppies, regardless of rearing history, have been shown to spontaneously exhibit the level of skill seen in adult dogs (Hare et al, 2002, Riedel et al 2008). Yet future work should replicate the current studies with young puppies of varying breeds to rule out the effect of their environment in producing the observed breed differences. The owners of working dogs may choose these breeds because they more than other owners enjoy cooperating and communicating with their dogs and correspondingly rear dogs better able to use human social cues. In addition, future tests should examine how robust the differences are between these same groups of 
dogs in other social skills. Such tests will elucidate what psychological system(s) the proposed second wave of selection acted upon during dog evolution. Did direct selection shape social-emotional systems, more complex cognitive abilities, or both?

Taken together, the current results provide direct evidence for the effect of domestication (i.e. selection against aggression and fear and for interest in humans; Belayev, 1979; Trut, 2001) on dog social problem solving skill. These results also suggest that a sub-group of modern dog breeds have been under direct selection for their ability to communicate in order to cooperate with humans. It is hoped then, as continued inter and intraspecific comparisons among canids are made, further inferences can be made regarding the evolutionary processes responsible for driving changes in social problem solving behavior in canids. Such inferences will have possible implications for how social problem-solving behaviors may have been selected in humans (Hare \& Tomasello, 2005).

\section{Acknowledgements}

We thank the New Guinea Singing Dog Conservation Society for sponsoring the travel of the first author to visit their facility. We also thank Julia Riedel, David Buttelmann, Katrin Schumann, Linda Schulz, and Linda Scheider for help in recruiting and testing the dogs. In addition, we appreciate all of the time the owners took in having their dogs participate in this study. The research of the second author (B.H.) is supported by a Sofja Kovalevskaja award received from The Alexander von Humboldt Foundation and the 
German Federal Ministry for Education and Research. These experiments complied with all laws of the countries in which they were performed. 


\section{References}

Agnetta, B., Hare, B. and Tomasello, M. (2000). Cues to food location that domestic dogs (Canis familiaris) of different ages do and do not use. Animal Cognition 3: $107-112$.

Belayev, D. (1979). Destabilizing Selection as a Factor in Domestication. Journal of Heredity 70: 301-308.

Christiansen, F., Bakken, M., and Braastad, B. (2001). Behavioural differences between three breed groups of hunting dogs confronted with domestic sheep. Applied Animal Behaviour Science 72: 115-129.

Cooper, J., Ashton, C., Bishop, S., West, R., Mills, D. and Young, R. (2003). Clever hounds: social cognition in the domestic dog (Canis familiaris). Applied Animal Behaviour Science 81: 229-244.

Coppinger, R. and Coppinger, L. (2001). Dogs: a Startling New Understanding of Canine Origin, Behavior, and Evolution. New York: Scribner.

Coppinger, R. and Schneider, R. (1995). Evolution of Working Dogs. In Serpell, J., ed. The Domestic Dog: its evolution, behaviour and interactions with people. Cambridge: Cambridge University Press, 21-47.

Hare, B., Call, J. and Tomasello, M. (1998). Communication of food location between human and dog (Canis familiaris). Evolution of Communication 2(1): 137-159.

Hare, B. and Tomasello, M. (1999). Domestic dogs (Canis familiaris) use human and conspecific social cues to locate hidden food. Journal of Comparative Psychology 113(2): 173-177. 
Hare, B., Brown, M., Williamson, C. and Tomasello, M. (2002). The domestication of social cognition in dogs. Science 298: 1634-1636.

Hare, B., Plyusnina, I., Ignacio, N., Schepina, O., Stepika, A., Wrangham, R. and Trut, L. (2005). Social Cognitive Evolution in Captive Foxes Is a Correlated By-Product of Experimental Domestication. Current Biology 15: 226-230.

Hare, B., and Tomasello, M. (2005). Human-like social skills in dogs? Trends in Cognitive Sciences 9(9): 439-444.

Hart, B. and Hart, L. (1985). Selecting pet dogs on the basis of cluster analysis of breed behavior profiles and gender. Journal of the American Veterinary Medical Association 186(11): 1181-1185.

Koler-Matznick, J. (2002). The origin of the dog revisited. Anthrozoos 15: 98-118.

Koler-Matznick, J., Brisbin, I., Feinstein, M. and Bulmer, S. (2003). An updated description of the New Guinea singing dog (Canis hallstromi, Troughton 1957). Journal of Zoology, London 261: 109-118.

Miklosi, A. (2008). Dog Behaviour, Evolution, and Cognition. Oxford: Oxford University Press.

Miklosi, A., Polgardi, R., Topal, J. and Csanyi, V. (1998). Use of experimenter-given cues in dogs. Animal Cognition 1: 113-121.

Miklosi, A., Pongracz, P., Lakatos, G., Topal, J., and Csanyi, V. (2005). A Comparative Study of the Use of Visual Communicative Signals in Interactions Between Dogs (Canis familiaris) and Humans and Cats (Felis catus) and Humans. Journal of Comparative Psychology 119: 179-186. 
Parker, H., Kim, L., Sutter, N., Carlson, S., Lorentzen, T., Malek, T., Johnson, G., DeFrance, H., Ostrander, E. and Kruglyak, L. (2004). Genetic structure of the purebred domestic dog. Science 304: 1160-1164.

Pongracz, P., Miklosi, A., Vida, V., Csanyi, V. (2005). The pet dog's ability for learning from a human demonstrator in a detour task is independent from the breed and age. Applied Animal Behaviour Science 90 (3-4): 309-323.

Riedel, J., Buttelmann, D., Call, J., and Tomasello, M. (2006). Domestic dogs (Canis familiaris) use a physical marker to locate hidden food. Animal Cognition 9(1): 27-35.

Riedel, J., Schumann, K., Kaminski, J., Call, J., and Tomasello, M. (2008). The early ontogeny of human-dog communication. Animal Behavior 75: 1003-1014.

Scott, J. and Fuller, J. (1965). Genetics and Social Behavior of the Dog. Chicago: The University of Chicago Press.

Soproni, K., Miklosi, A., Topal, J. and Csanyi, V. (2001). Comprehension of human communicative signs in pet dogs (Canis familiaris). Journal of Comparative Psychology 115(2): 122-126.

Soproni, K., Miklosi, A., Topal, J. and Csanyi, V. (2002). Dogs' (Canis familiaris) responsiveness to human pointing gestures. Journal of Comparative Psychology 116(1): 27-34.

Trut, L. (2001). Experimental studies of early canid domestication. In Ruvinsky, A. and J. Sampson, ed. The Genetics of the Dog. New York: CABI Publishing, 15-42. 
Udell, M., Dorey, N. and Wynne, C., 2008. Wolves outperform dogs in following human social cues. Animal Behaviour 76: 1767-1773.

Vila, C., Savolainen, P., Maldonado, J., Amorim, I., Rice, J., Honeycutt, R., Crandall, K., Lundeberg, J. and Wayne, R. (1997). Multiple and ancient origins of the domestic dog. Science 276: 1687-1689.

Viranyi, Z., Gasci, M., Kubinyi, E., Topal, J., Belenyi, B., Ujfalussy, D., and Miklosi, A. (2008). Comprehension of human pointing gestures in young human-reared wolves (Canis lupus) and dogs (Canis familiaris). Animal Cognition 11: 373-387.

Wilsson, E. and Sundgren, P. (1997). The use of a behaviour test for the selection of dogs for service and breeding, I: Method of testing and evaluating test results in the adult dog, demands on different kinds of service dogs, sex and breed differences. Applied Animal Behaviour Science 53: 279-295. 
Figure 1 Test setup. The experimenter sat equidistant between the two bowls, 1.5 meters from where the dog sat at a pre-determined point (delineated by the tape markings on the floor). Here, the experimenter is giving the Gaze cue (Experiment 2).

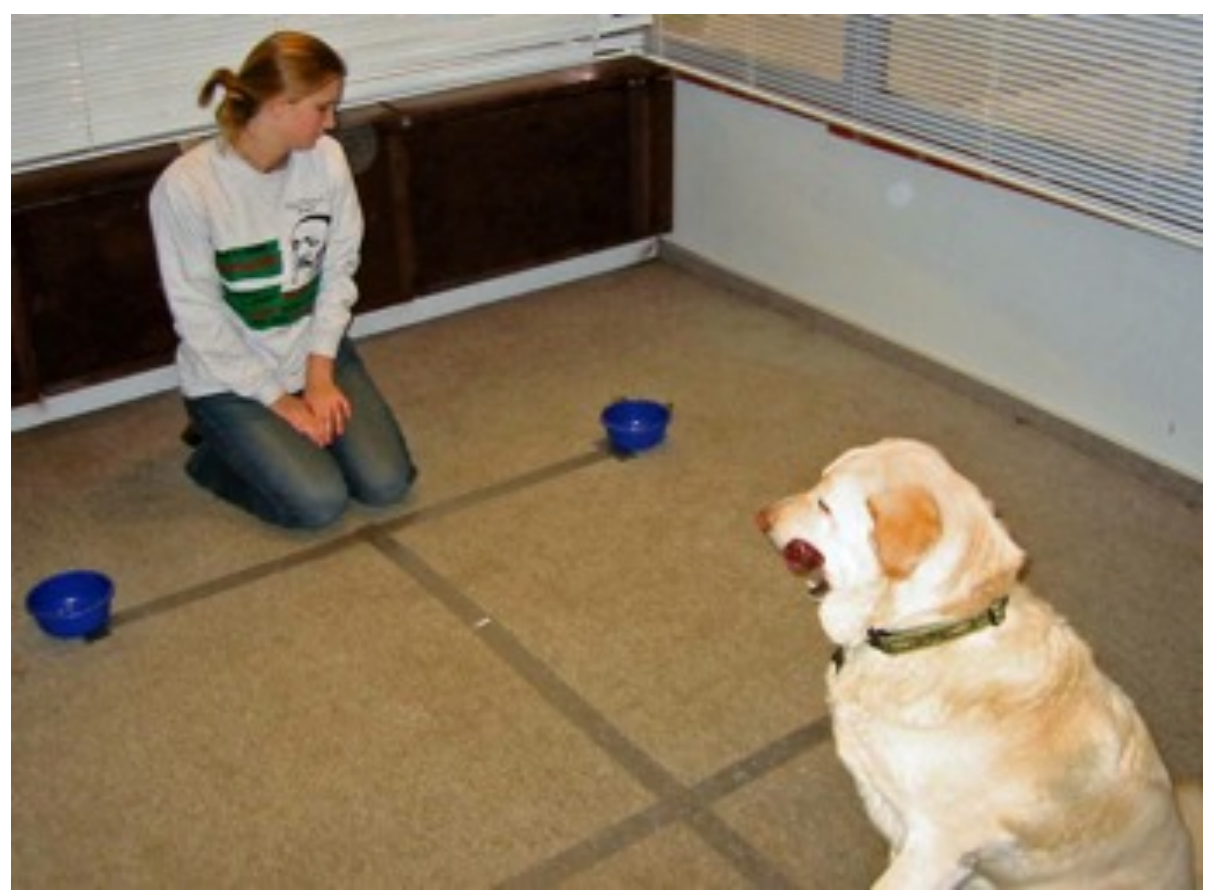


Figure 2 The number of correct choices (+SEM) out of eighteen trials with each of the different social cues by New Guinea Singing Dogs and working/companion breeds $\left({ }^{*} \mathrm{p}<0.05,{ }^{* *} \mathrm{p}<0.01, * * * \mathrm{p}<0.001\right.$ one-sample t-test significance) in Experiment 1.

Significant differences between groups using independent-samples t-tests are shown.

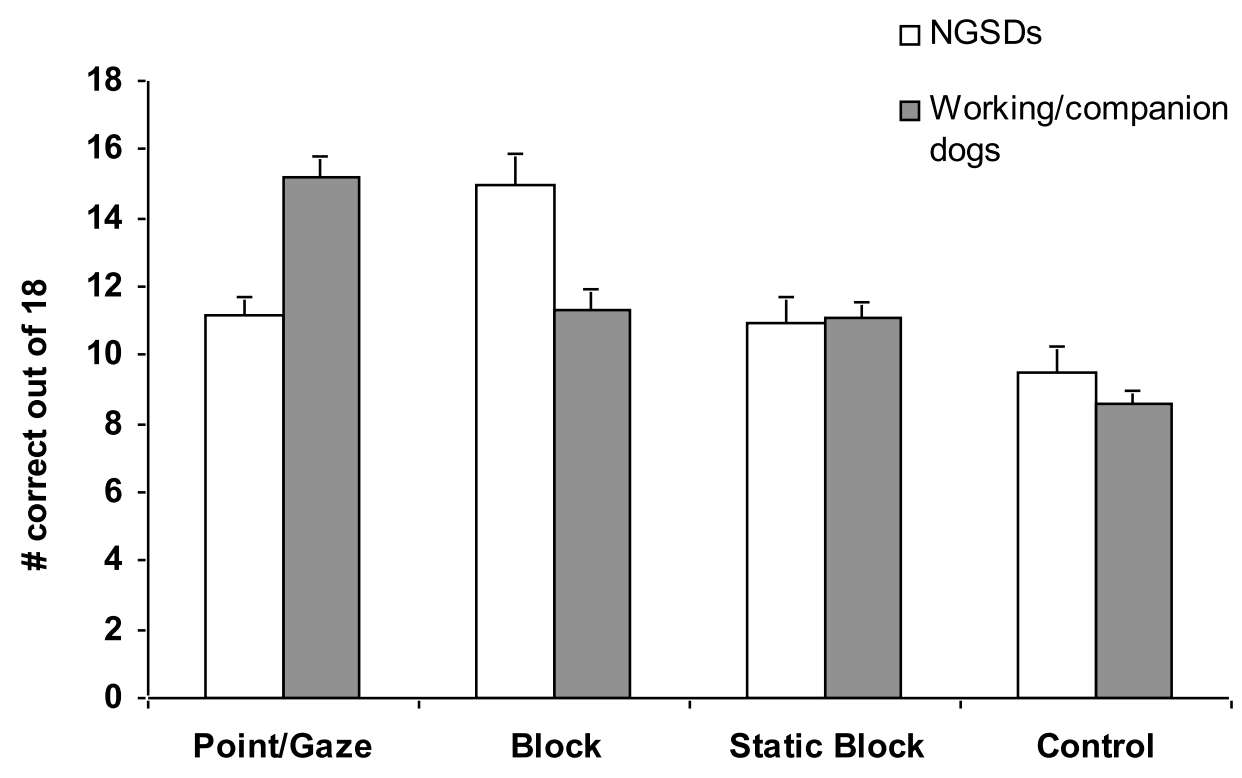


Figure 3 The number of correct choices (+SEM) out of eighteen trials with each of the different social cues by each of the four breed groups tested $\left(* \mathrm{p}<0.05,{ }^{*} \mathrm{p}<0.01\right.$, $* * * \mathrm{p}<0.001$, one-sample t-test) in Experiment 2. Huskies represent the wolf-like working breed, shepherds the non wolf-like working breed, basenjis the wolf-like non-working breed, and toy poodles the non wolf-like non-working breed.

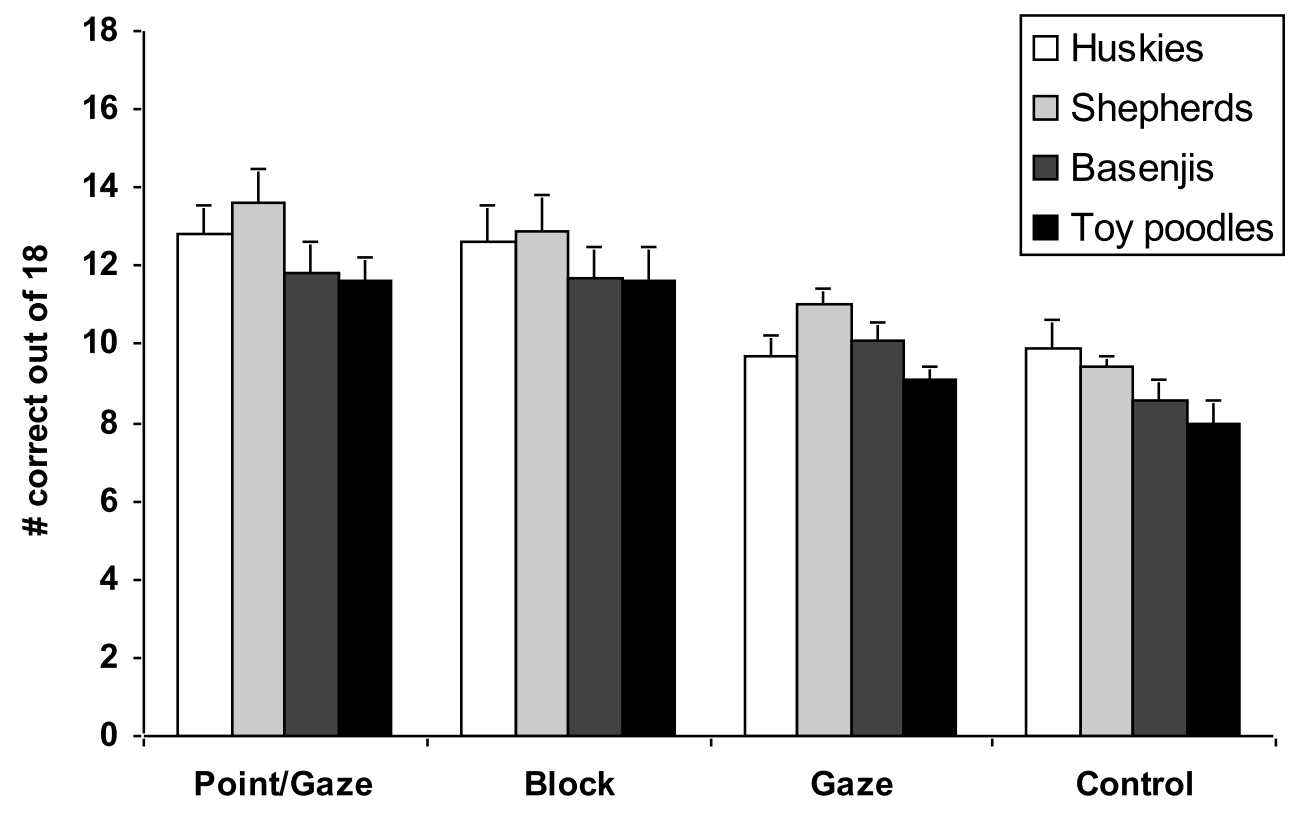


Figure 4 The percentage of individuals in Experiment 2 among the working dogs

(shepherds and huskies) and non-working dogs (toy poodles and basenji) that found the hidden food above chance levels with at least one and two of the communicative cues provided by the human experimenter. Significant differences between groups using $\chi^{2}$ Fisher's exact test are shown.

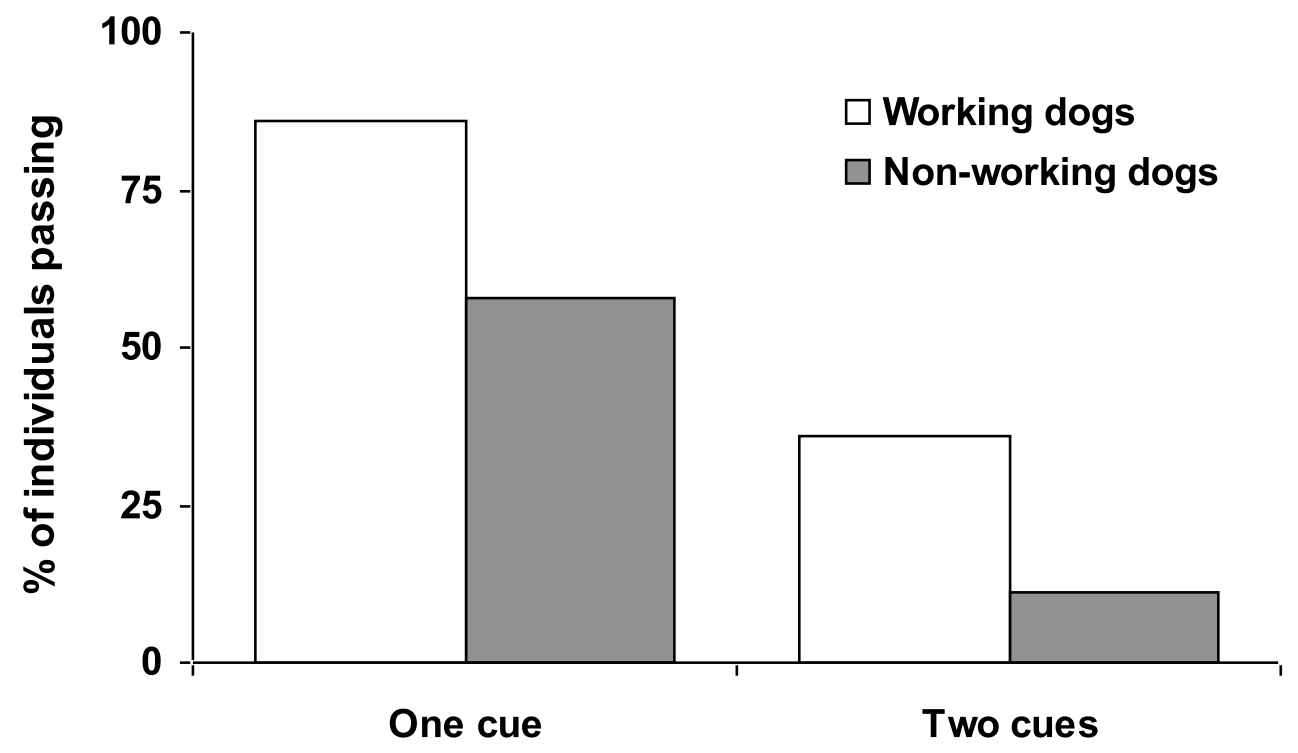


Figure 5 The percentage of individuals in Experiment 2 among the wolf-like dogs (huskies and basenji) and non wolf-like dogs (shepherds and toy poodles) that found the hidden food above chance levels with at least one and two of the communicative cues provided by the human experimenter. There were not significant differences between groups in the proportion of individuals successful in either measure (Fisher's exact test).

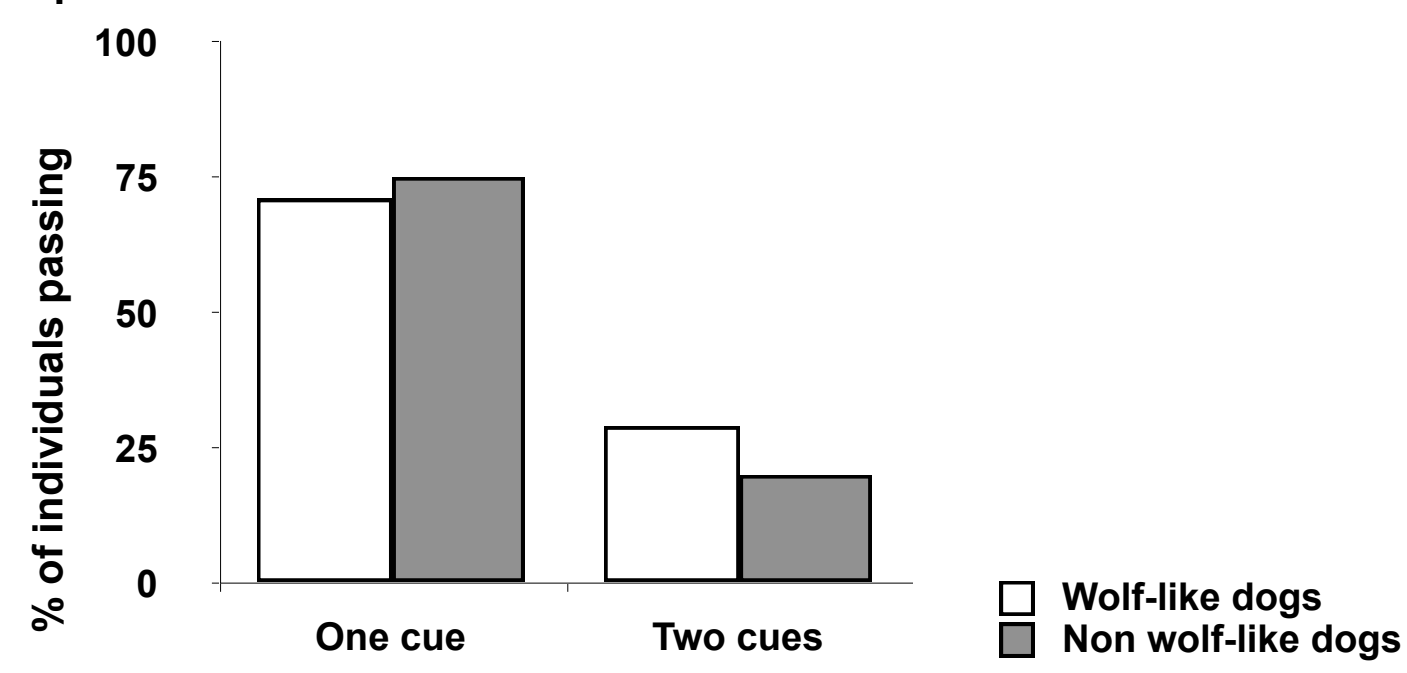

